Norbert Rogge* und Thomas Fröhlich

\title{
Nutzung eines kommerziellen EMK-Systems als Bestandteil einer Kibble-Waage
}

\author{
Utilization of a commercial EMFC system as component in a Kibble balance
}

DOI 10.1515/teme-2021-0053

Zusammenfassung: Eine Planck-Waage ist eine einfach bedienbare und vergleichsweise kostengünstige Umsetzung des Kalibrierprinzips einer Kibblewaage. Zu diesem Zweck besteht das Gesamtsystem möglichst aus kommerziell verfügbaren Komponenten wie z.B. einer Wägezelle nach dem Prinzp der elektromagnetischen Kraftkompenastion. Diese dient sowohl der Aufnahme des Masseprüflings im Wägemodus, als auch zur Führung im Bewegungsmodus. Da Abweichungen vom Idealverhalten bezüglich Kraftund Momentenaufnahme sowie der Bewegungstrajektorie großen Einfluss auf das Messergebnis haben, müssen diese analysiert und im Messprozess berücksichtigt werden.

Schlüsselwörter: Planck-Waage, Kibble-Waage, Kalibrierung, Führungsabweichungen, Elektromagnetische Kraftkompenastion

Abstract: A Planck-Balance is an easy to use and relatively cost-efficient realization of the calibration method of a Kibble balance. For this purpose, the system preferably consists of commercially available components e.g. an electromagnetic force compensated load cell (EMFC). This load cell acts as load carrier for the mass specimen in the force mode as well as guiding system in the velocity mode. Since deviations from an ideal behavior regarding the transfer of torques and forces and the motion trajectory have a great impact on the measurement result, these deviations must be characterized and incorporated into the analysis of the measurement data.

Keywords: Planck-Balance, Kibble balance, calibration, guiding deviations, electromagnetic force compensation

\footnotetext{
*Korrespondenzautor: Norbert Rogge, TU Ilmenau, Fachgebiet Prozessmesstechnik, IImenau, Deutschland, e-mail: norbert.rogge@tu-ilmenau.de

Thomas Fröhlich, TU Ilmenau, Fachgebiet Prozessmesstechnik, Ilmenau, Deutschland, e-mail: thomas.froehlich@tu-ilmenau.de
}

\section{Einführung}

Eine Methode, die zur Bestimmung des Wertes des Planckschen Wirkungsquantums im Zuge Neudefinition des Kilogramm verwendet wurde, ist die sogenannte Kibble-Waage (ehemals Watt-Waage) [3, 4, 7, 9, 10, 15], welche nach der im Mai 2019 erfolgten Neudefinition als alternative Variante zur Kalibrierung in der Kraftmess- und Wägetechnik nutzbar ist. Ziel der Kalibrierung einer Waage nach dem Prinzip der elektromagnetischen Kraftkompensation (EMK) ist im Wesentlichen die Bestimmung der Aktorkonstante $B l$ des Tauchspulenaktors, welcher die Kompensationskraft mit Hilfe des Kompensationsstroms $I$ erzeugt. Bei der etablierten Kalibrierung mit Massenormalen, bei der die Aktorkonstante $B l$ durch die Gewichtskraft $F$ einer bekannten Masse $m$ bestimmt wird, gilt:

$$
F=m \cdot g=B l \cdot I=\frac{B l \cdot U_{R}}{R} .
$$

Der Kompensationsstrom wird dabei als Spannungsabfall $U_{R}$ über einem kalibrierten Messwiderstand $R$ ermittelt. In einer Kibble-Waage wird das $B l$ im Gegensatz dazu in einem zweiten Betriebsmodus bestimmt, in dessen Verlauf die Spule relativ zum Magnetfeld bewegt wird. Aus dem Verhältnis von induzierter Spannung $U_{\text {ind }}$ und Spulengeschwindigkeit $v$ kann das $B l$ mit

$$
B l=\frac{U_{\text {ind }}}{v}
$$

bestimmt werden. Durch Kombination der beiden Betriebsmodi kann die Masse unter Kenntnis der lokalen Fallbeschleunigung $g$ ohne die Rückführung auf ein Massenormal als

$$
m=\frac{U_{\text {ind }} \cdot U_{R}}{R \cdot g \cdot v}
$$

bestimmt werden.

\section{Die Planck-Waage}

Die Planck-Waage wurde in einem Kooperationsprojekt zwischen der Physikalisch-Technischen Bundesanstalt 
(PTB) Braunschweig und dem Institut für Prozessmessund Sensortechnik (IPMS) der Technischen Universität Ilmenau entwickelt. Um eine möglichst kostengünstige Umsetzung der Kibble-Kalibrierung realisieren zu können, werden nach Möglichkeit kommerziell verfügbare Teilsysteme eingesetzt. Im sogenannten PB2-System, welches für die Kalibrierungen in der Genauigkeitsklasse E2 gemäß OIML R111 [8] in einem Bereich von $1 \mathrm{mg}$ bis $100 \mathrm{~g}$ konzipiert ist, dient eine Wägezelle vom Typ WZA215 der Firma Sartorius [14] als Grundlage.

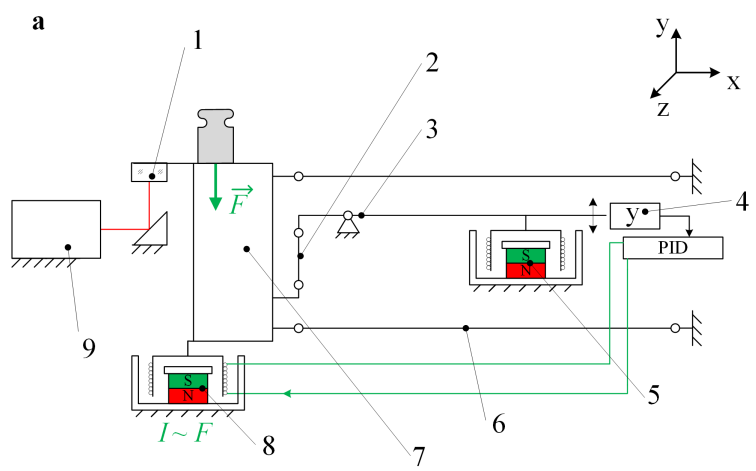

b

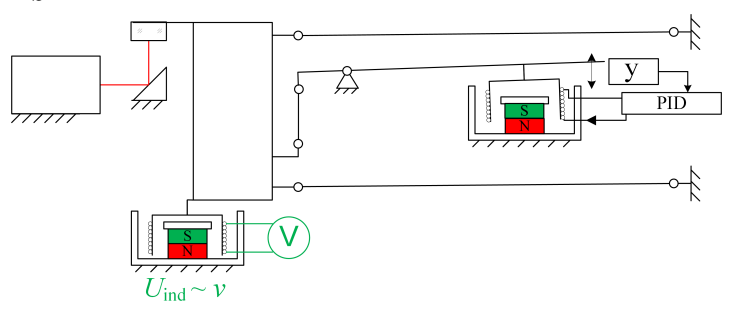

Abb. 1: Schema der Planck-Waage (PB2-System) im (a) Wägemodus und (b) Bewegungsmodus. 1 - Messspiegel, 2 - Koppelband, 3 - Übertragungshebel, 4 - Positionssensor, 5 - Antriebsaktor, 6 - Parallellenker, 7 - Lastträger, 8 - Messaktor, 9 - Interferometer.

An den Lastträger dieser Wägezelle wird, wie in Abbildung 1 schematisch dargestellt, ein weiterer Tauchspulenaktor angebracht, dessen Spule (Messspule) als die eigentliche Spule für das Kibbleexperiment dient. Zur rückführbaren Bestimmung der Bewegung der Messspule wird an den Lastträger außerdem ein Messspiegel befestigt, dessen Verschiebung mit Hilfe eines kommerziell verfügbaren Homodyninterferometers [16] erfasst wird. Das verwendete Differenzinterferometer hat den Vorteil, dass der Referenzspiegel, wie in Abbildung 2 dargestellt, gestellfest neben der Nulllage des Messpiegel befestigt werden kann, wodurch die Totstrecke der interferometrischen Messung minimiert wird und damit Brechzahleinflüsse gering werden. Im Wägemodus nimmt die Parallelfederführung der
Wägezelle parasitäre Kräfte und Momente auf und dient so der Reduktion von Ecklastfehlern. Im Bewegungsmodus dient sie als Führung für die eingeprägte Auslenkung der Messspule, während der interne Aktor (Antriebsaktor) der EMK-Wägezelle die erforderliche Kraft für die Bewegung erzeugt. Im Wägemodus wird der Antriebsaktor außerdem verwendet, um den Arbeitspunkt des Messaktors so einzustellen, dass dessen Kompensationsstrom im belasteten und unbelasteten Zustand den gleichen Betrag jedoch unterschiedliche Vorzeichen aufweist. Dies dient der Verringerung des sogenannten Spulenstromeffektes [5, 15]. Dieser Effekt beschreibt die Veränderung der Aktorkonstante $B l$ durch die Überlagerung des Magnetfeldes des Aktors mit dem von der bestromten Spule erzeugten Magnetfeld. Durch die Betragsgleicheit und die Vorzeichenumkehr im be- und entlasteten Zustand wird die Auswirkung dieses Effektes kompensiert [13].

Aufgrund der eingeschränkten Auslenkung, die mit der Mechanik der EMK-Wägezelle erzeugt werden kann, ist es im PB2-System nicht möglich, das Bewegungsexperiment wie bei bisher aufgebauten Kibble-Waagen mit konstanter Geschwindigkeit über mehrere Millimeter durchzuführen. Stattdessen wird die Spule harmonisch angeregt und die Amplituden von $U_{\text {ind }}$ und $v$ mit einem Sinus-Fit $[4,6]$ bestimmt. Dies erlaubt auch bei eingeschränktem Bewegungsbereich eine lange Integrationszeit für die Bestimmung der Aktorkonstante $B l$. Da die Amplitude der Geschwindigkeit $v$ aus der vom Interferometer ermittelten Verschiebung des Lastträgers in y-Richtung ermittelt wird, müssen die Signale mit einer auf die SI-Einheitendefinition zurückgeführten Zeitbasis erfasst werden. Dies wird im PB2-System sichergestellt, indem das Signal zur Triggerung der Erfassung der einzelnen Messwerte unter Zuhilfenahme eines GPS disziplinierten ofenstabilsierten Quarzoszillators (OCXO) erzeugt wird [17].

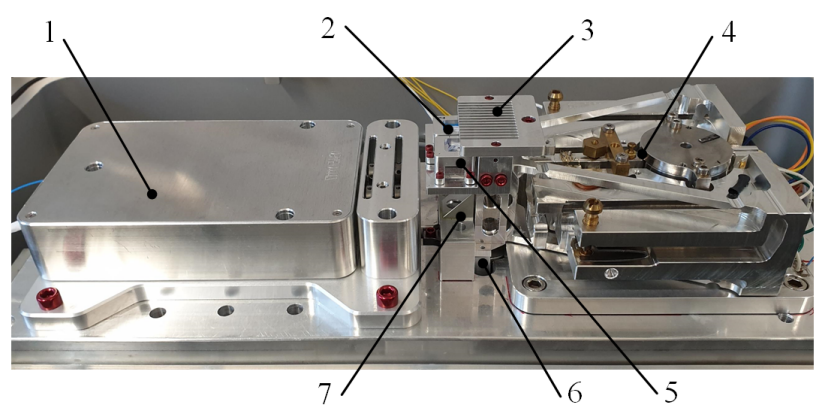

Abb. 2: Foto der Planck-Waage (PB2-System), Breite ca. 300 mm, 1 - Interferometer, 2 - Referenzspiegel, 3 - Waagschale, 4 - Wägezelle, 5 - Messspiegel, 6 - Halterung Messspule, 7 - Umlenkspiegel. 
Die Erzeugung des Anregungssignals und die Regelung der Hebelposition in beiden Betriebsmodi werden auf einem digitalen Signalprozessor implementiert, dessen Ein- und Ausgänge jeweils über eine Auflösung von 16 bit verfügen und mit geeigneter Verstärkungs- und Signalaufbereitungselektronik versehen sind. Aufgrund der Rückkopplung im Regelkreis, der Trägheit der Mechanik und der Induktivität der Aktorspule weist der Kompensationstrom eine Schwankungsbreite auf, die deutlich kleiner als die nominelle Auflösung des DSP-Systems ist. Dieser Kompensationsstrom im Wägemodus und die induzierte Spannung im Bewegungsmodus werden mit einem hochauflösenden, mit Hilfe eines Josphsonnormals kalibrierten Multimeter vom Typ Keysight 3458A gemessen. Die unterschiedlichen Verbindungen der Mess- und Antriebsspule zum Mess- und Regelungssystem in den beiden Betriebsmodi werden automatisiert durch eine selbst entwickelte Umschalteinheit hergestellt, die auf thermospannungsarmen Relais mit guter thermischer Kopplung zueinander basiert. Zur Erleichterung des Sinus-Fits wird außerdem die Schleifenfrequenz des implementierten Reglers inklusive des Signalgenerators mit einem auf den OCXO rückgeführten Frequenzzähler bestimmt. Dies ermöglicht die Korrektur der vom Nutzer vorgegebenen Anregungsfrequenz um die von der Zeitbasis des DSP erzeugten Abweichungen. Dadurch ist die tatsächliche Anregungsfrequenz mit geringer Unsicherheit bekannt, was die Bestimmung von Amplitude und Phase der im Bewegungsmodus erfassten Signale erleichtert $[4,6]$.

\section{Führungsabweichungen}

\subsection{Erzeugung der Bewegung}

Im PB2-System werden der Antriebsaktor und der interne Positionssensor [2] der Waage genutzt, um die sinusförmige Auslenkung der Messspule zu erzeugen. $\mathrm{Zu}$ diesem Zweck wird ein Regler implementiert, dessen Eingang die Hebelposition und dessen Aushang der Spulenstrom des Antriebsaktors ist. Durch Vorgabe einer sinusförmigen Sollposition mit festgelegter Frequenz kann dann eine Anregung vorgeben werden, deren Amplitude weitestgehend unabhängig von den veränderlichen Eigenschaften des Antriebsaktors ist. Durch eine vorhergehende statische Kalibrierung und Linearisierung des Positionssensors mit Hilfe des Interferometers werden die Verzerrungen des Anregungssignals reduziert.

Da das Hebelsystem, welches die Anregung auf das Koppelstück der Parallelfederführung überträgt, eine be-

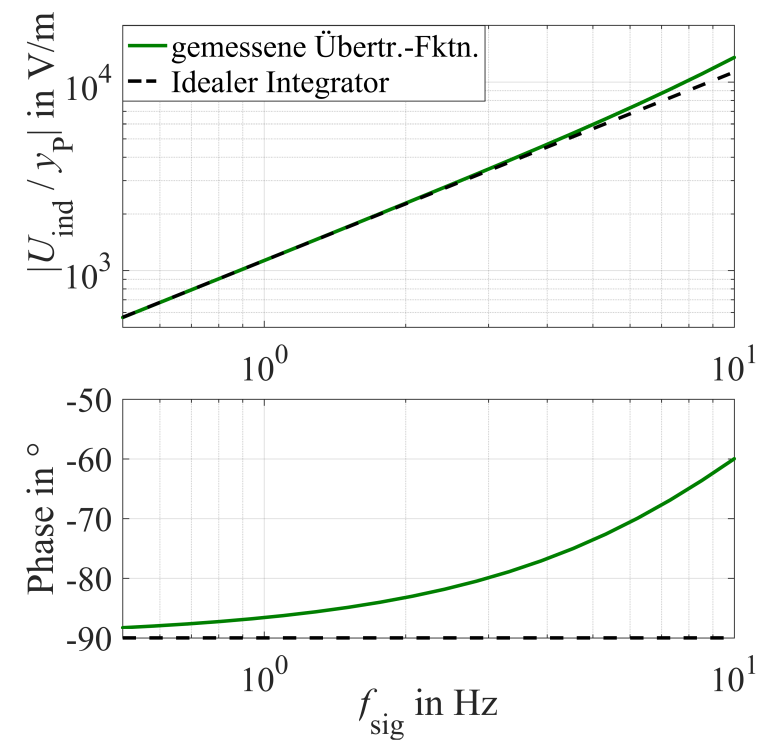

Abb. 3: Experimentell ermittelter Amplitudenfrequenzgang und ermittelte Phase der Übertragungsfunktion der induzierten Spannung bei Anregung am Waagenhebel.

grenzte Steifigkeit aufweist, wird dessen in Abbildung 3 dargestellte Übertragungsfunktion identifiziert. Hierzu werden die induzierte Spannung in der Messspule und das Signal des Positionssensors synchron erfasst während die Waage harmonisch angeregt wird. Bei vernachlässigbarer Steifigkeit des Übertragungssystems, sollte diese Übertragungsfunktion einen idealen Integrator darstellen. Aus der Differenz von idealem Integrator und dem experimentell bestimmten Verhältnis kann dann die Übertragungsfunktion des Anregungssystems bestimmt werden. Mit dieser ist eine Korrektur möglich, die eine konstante Anregungsamplitude des Koppelstücks und der daran befestigten Messspule unabhängig von der Anregungsfrequenz sicherstellt. Dadurch kann auch bei variierender Frequenz mit einer konstanten Amplitude angeregt werden, was genutzt werden kann, um verschiedene frequenzabhängige Einflüsse auf die Bestimmung der Aktorkonstante $B l$, wie beispielsweise die in Abschnitt 3.3 beschriebenen Gestellschwingungen, zu identifizieren.

\subsection{Statische Abweichungen}

Die Umsetzung der Kibblekalibrierung erfordert eine präzise Ausrichtung der Vorzugsachse der Parallelfederführung an der Fallbeschleunigung $g$. Bei der konventionellen Kalibrierung ist dies weniger kritisch, solange die Ausrichtung bei der Messung und der Kalibrierung mit einem Masse- 
normal die gleiche ist, was insbesondere bei EMK-Waagen mit internem Justiergewicht gilt.

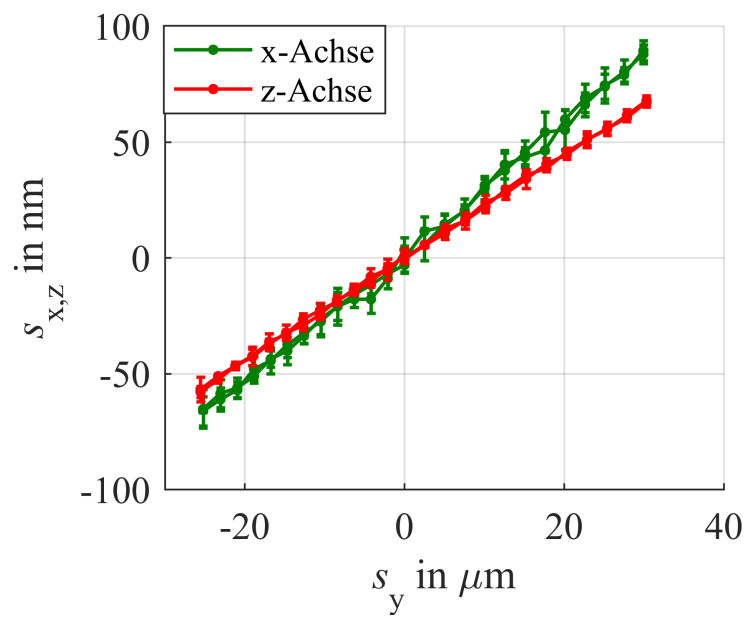

Abb. 4: Horizontale Verschiebung $s_{x, y}$ des Messspiegels bei Auslenkung $s_{y}$ des Lastträgers; Koordinatenrichtungen wie in Abb. 1.

Da diese Achse nicht geometrisch fassbar ist, wird sie im PB2-System mit Hilfe eines kalibrierten Spiegelquaders und eines weiteren Interferometers bestimmt, welches die in Abbildung 4 dargestellte horizontale Verschiebung des Quaders misst. Die dargestellten Verschiebungen entsprechen Winkelabweichungen von 2,2 mrad bzw. 2,8 mrad zwischen der Messachse des internen Interferometers des PB2-Systems und der Bewegegungsachse der Parallelfederführung. Die Rechtwinkligkeit der Spiegelflächen des Quaders ist laut Kalibrierung besser als 21,8 $\mu \mathrm{rad}$ und der Messstrahl muss besser als 0,23 mrad mit Flächennormalen übereinstimmen, damit ein stabiles Interferometersignal erfasst werden kann. Somit ist der Winkel zwischen der Spiegeloberfläche des Quaders mit ausreichend geringer Unsicherheit bekannt und mit Hilfe eines Flüssigkeitsspiegels und einem Autokollimationsfernrohr kann die Achse parallel zur Richtung der lokalen Fallbeschleunigung justiert werden. Der resultierende relative Unsicherheitsbeitrag für die Bestimmung der Aktorkonstante $B l$ beträgt $1,2 \cdot 10^{-6}$.

Im Wägemodus des PB2-Systems wird die Masse des Prüfgewichts aus der Differenz des Kompensationstroms des Messaktors im be- und entlasteten Zustand bestimmt. Da sich die Aktorkonstante $B l$ des Messaktors mit $0,47 \cdot 10^{-6} / \mu \mathrm{m}$ bei Verschiebung in y-Richtung ändert, muss diese in den beiden Phasen des Wägemodus möglichst übereinstimmen. Aufgrund der elastischen Eigenschaften des Übertragungshebels und des Koppelbandes, werden mit dem Interferometer bei Regelung mit

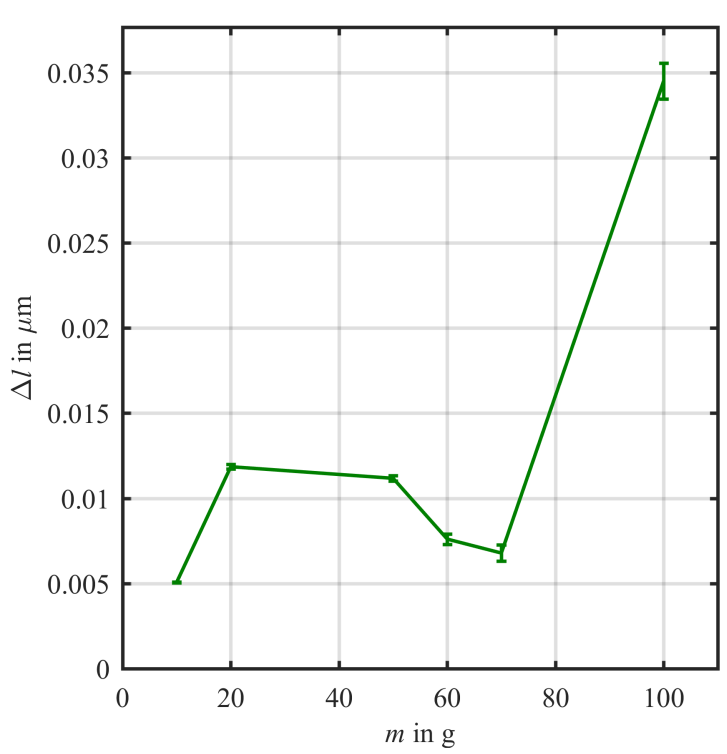

Abb. 5: Differenz $\Delta l$ der Auslenkung des Messspiegels bei Lastwechsel in Abhängigkeit von der Masse $m$ auf der Waagschale.

Hilfe des internen Positionssensors der Waage die in Abbildung 5 dargestellten Abweichungen von bis $\mathrm{zu} 35 \mathrm{~nm}$ gemessen. Die große Nichtlinearität dieser Verschiebungsdifferenzen in Abhängigkeit von der Prüfmasse ist auf eine Überlagerung der Koppelbandverformung und der Verformung des Parallelführungssystems zurückzuführen [18]. Aus dieser Positionierunsicherheit ergibt sich ein relativer Unsicherheitsbeitrag von $0,016 \cdot 10^{-6}$ für die Ermittlung der Aktorkonstante $\mathrm{Bl}$.

\subsection{Dynamische Abweichungen}

Aufgrund von Bauraumbeschränkungen ist es derzeit im PB2-System nicht möglich, die Messachse des Interferometers fluchtend zur Mitte der Spule anzuordnen. Bei einer Verkippung des Lasträgers hat dies zur Folge, das die Messung der Spulenverschiebung und somit auch der Geschwindigkeit mit einem Abbefehler behaftet ist.

Zur Identifikation der bei Auslenkung der Messspule auftretenden Kippschwingungen wird die Bewegung des Lastträgers mit einem Dreistrahlinterferometer ermittelt, während das System mit einem Chirp-Signal angeregt wird [11]. Aus den Verschiebungen des Lastträgers an drei Punkten, deren Abstände zueinander bekannt sind, werden dann die Auslenkung und die Kippung um zwei Achsen bestimmt.

Bei sehr langsamer Auslenkung des Hebels treten bei der verwendeten Wägezelle keine signifikanten Ver- 


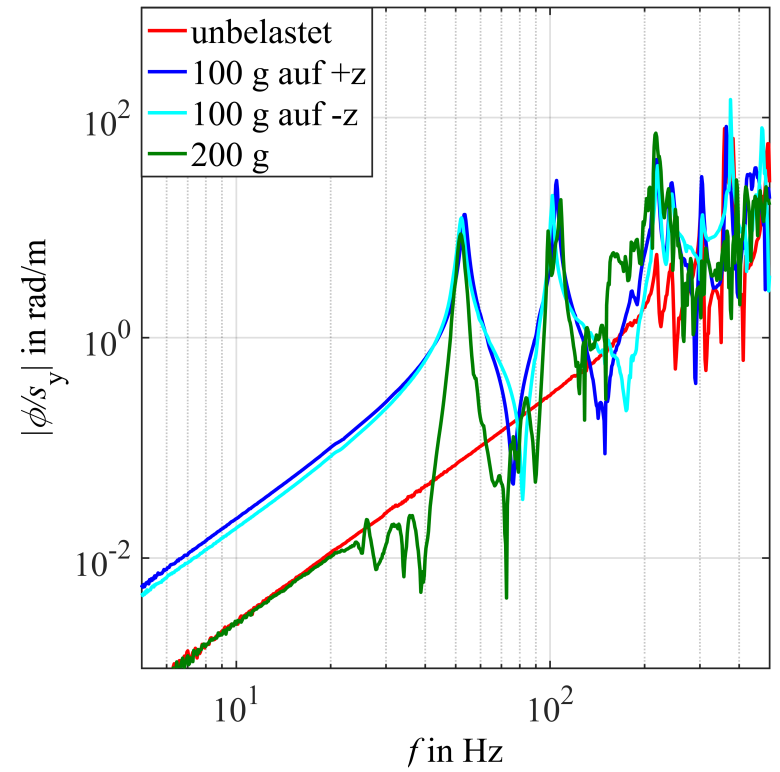

Abb. 6: Experimentell bestimmter Amplitudenfrequenzgang der Übertragungsfunktion der Schwingungen des Lastträgers um die $x$-Achse bei Anregung einer Vertikalbewegung.

kippungen des Koppelstücks auf Grund der Anregung auf [11], jedoch steigen die Schwingungsamplituden mit zunehmender Frequenz stark an. Die in Abbildung 6 gezeigten Kippschwingungen um die $\mathrm{x}$-Achse sind als Übertragungsfunktion mit der Lastträgerbewegung als Eingangsgröße dargestellt, da bei Auslenkungen von weniger als $40 \mu \mathrm{m}$ die Kippwinkel linear von dieser Auslenkung abhängen. Im unteren Frequenzbereich steigen die beobachtbaren Amplituden linear mit der Frequenz an, bis bei etwa $50 \mathrm{~Hz}$, je nach Belastungszustand, mechanische Resonanzen auftreten. Analoge Effekte werden auch bei Betrachtung der Kippschwingungen um die z-Achse beobachtet. Als Belastungsfälle wurde das geprüfte System in einem Abstand von etwa $50 \mathrm{~mm}$ zur Bewegungsebene des Hebels mit $100 \mathrm{~g}$ belastet. Diese unsymmetrische Belastung wurde auf beiden Seiten untersucht und hat in beiden Fällen eine starke Erhöhung der Winkelamplituden und eine Reduzierung der Resonanzfrequenz zur Folge. Eine ähnliche Resonanzfrequenz wird auch erreicht, wenn beide Aufsetzpunkte mit jeweils $100 \mathrm{~g}$ belastet werden, jedoch sind die Amplituden im niedrigen Frequenzbereich ähnlich zu denen im unbelasteten Zustand. Daher ist beim Einsatz der Wägezelle in einer Kibble-Waage auf eine möglichst symmetrische Lastverteilung zu achten.

Um die erreichbaren Unsicherheiten des PB2-Systems weiter reduzieren zu können, wurden Untersuchungen zu den Ursachen der Verkippungen durchgeführt. Die beobachteten Schwingungen entstehen einerseits durch eine
Verformung des Parallelfedersystems der Wägezelle, da deren Festkörpergelenke Fertigungstoleranzen unterliegen und der Schwerpunkt der Masse des Lasträgers und dessen Anbauten nicht fluchtend zum Kraftangriffspunkt der Anregung liegt. Eine weitere Komponente stellt die Verkippung der Wägezelle um ihre Befestigung im Gesamtsystem dar. Dieser Effekt wird untersucht, indem der Messspiegel des in den vorhergehenden Untersuchungen genutzten Dreistrahlinterferometers nun auf den gestellfesten Teil der monolithisch aufgebauten Wägezelle befestigt wird. In dieser Konfiguration können zwar die gleichen Kippwinkel, aber nicht mehr die Verschiebung des Lastträgers gleichzeitig gemessen werden. Zur Bestimmung der Übertragungsfunktion wird daher der Lasträger mit einem Chirpsignal mit konstanter Amplitude angeregt und die Einhüllenden der resultierenden Chirpsignale im Verlauf der Kippwinkel des Gestells durch einen Polynomfit ermittelt [1]. Wie in Abbildung 7 zu erkennen ist, führt dies im Gegensatz zur Ermittlung der Übertragungsfunktion aus dem Verhältnis von Kippwinkel zur Verschiebung des Lasträgers zu einem glatteren Verlauf der Übertragungsfunktion, bei dem jedoch nicht alle Resonanzphänomene aufgelöst werden können. Für den dargestellten Frequenzbereich stellt die Kippschwingung des Gestells einen signifikanten Anteil an der am Lastträger auftretenden Gesamtschwingung dar.

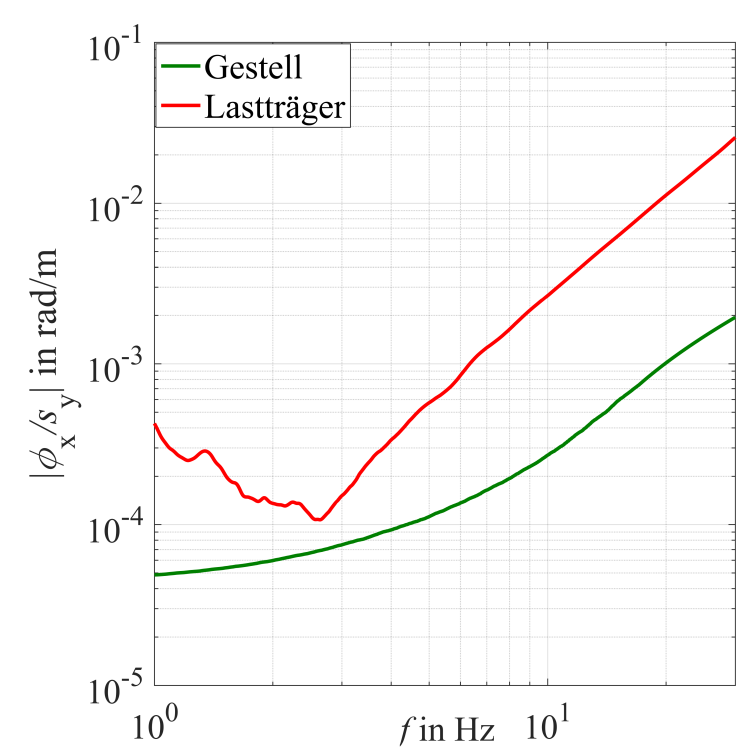

Abb. 7: Experimentell bestimmter Amplitudenfrequenzgang der Übertragungsfunktion der Schwingungen des Gestells um die $x$-Achse bei Anregung einer Vertikalbewegung.

Im Bewegungsmodus des PB2-Systems wird mit einer Frequenz von $4 \mathrm{~Hz}$ und einer Amplitude von $20 \mu \mathrm{m}$ ange- 
regt, was Kippschwingungen mit Amplituden von bis zu $100 \mu \mathrm{rad}$ zur Folge hat. Die resultierenden relativen Fehler bei der Bestimmung der Aktorkonstante $B l$ von etwa $32 \cdot 10^{-6}$ können jedoch nach einmaliger Bestimmung der Amplituden korrigiert werden [12].

\section{Zusammenfassung}

Beim Einsatz kommerziell verfügbarer EMK-Wägezellen ergeben sich Vorteile bei den Kosten des Gesamtsystems. Jedoch muss das dynamische Verhalten der Wägezelle detaillierter betrachtet werden als es bei dessen üblichen, statischem Einsatz notwendig wäre. Die Untersuchungen zeigen, dass nicht nur die Verformung der eigentlichen Waagenmechanik eine Rolle spielt, sondern auch die Verformung an der unteren Befestigungsstelle der Wägezelle. Eine Charakterisierung dieser Eigenschaften und daraus abgeleitete Korrekturen des Messergebnisses sowie die Berücksichtigung des Spulenstromeffektes [5, 15] ermöglichen jedoch relative Unsicherheiten in der Größenordnung von wenigen $10^{-6}$.

Danksagung: Die Autoren danken dem Bundesministerium für Bildung und Forschung BMBF für die Förderung des Projekts 03VP02581 „PLANCK-WAAGE - Selbstkalibrierende Präzisionswaagen für den industriellen Einsatz" im Programm Validierung des technologischen und gesellschaftlichen Innovationspotenzials - VIP + und der VDI/VDE Innovation + Technik GmbH für administrative Begleitung, sowie den Projektpartnern an der PTB und der TU Ilmenau für die fachlichen Diskussionen.

\section{Literatur}

[1] H. Bai, N. Rogge, C. Rothleitner und T. Fröhlich. Model based correction of motion deviations in the planck-balance. In 20. GMA/ITG-Fachtagung Sensoren und Messsysteme, Nürnberg, Germany, S. 554-560. AMA Verband für Sensorik und Messtechnik e.V., 2019. 10.5162/sensoren2019/6.4.5.

[2] C. Diethold, T. Fröhlich, F. Hilbrunner und G. Jäger. High precission optical position sensor for electromagnetic force compensated balances. In Proceedings of the IMEKO 21st TC3 International Conference, 2010.

[3] H. Fang, F. Bielsa, A. Kiss, T. Lavergne, Y. F. Lu, L. Robertsson, E. de Mirandés, S. Solve und M. Stock. Progress on the BIPM watt balance. In 2016 Conference on Precision Electromagnetic Measurements (CPEM 2016), S. 1-2, 2016. 10.1109/CPEM.2016.7540540.

[4] F. Hilbrunner, I. Rahneberg und T. Fröhlich. Wattwaage mit Hebelübersetzung auf Basis eines kommerziellen EMK-
Wägesystems. Technisches Messen, 2017. 10.1515/teme2017-0065.

[5] S. Li, F. Bielsa, M. Stock, A. Kiss und H. Fang. Coil-current effect in kibble balances: analysis, measurement, and optimization. Metrologia, 55(1):75-83, 2017. 10.1088/16817575/aa9a8e.

[6] S. Lin, C. Rothleitner, N. Rogge und T. Fröhlich. Influences on amplitude estimationusing the three-parameter sine fitting algorithm in the velocity mode of the planckbalance. Acta IMEKO, 9(3):40-46, sep 2020. 10.21014/acta_imeko.v9i3.781.

[7] R. R. Marangoni, J. Schleichert, I. Rahneberg, F. Hilbrunner und T. Fröhlich. A self-calibrating multicomponent force/torque measuring system. Measurement Science and Technology, 29(7):074002, 2017. 10.1088/1361-6501/aac00f. URL http://nbn-resolving.de/urn:nbn:de:gbv:ilm1-2017iwk-081:5.

[8] OIML R 111-1. International recommendation oiml $r$ 111-1, 2004. URL https://www.oiml.org/en/files/pdf_r/r111-1e04.pdf.

[9] A. Picard, M. Stock, H. Fang, T. Witt und D. Reymann. The bipm watt balance. IEEE Trans. Instrum. Meas., 56(2): 538-542, 2007. 10.1109/tim.2007.890783.

[10] I. A. Robinson und S. Schlamminger. The watt or kibble balance: a technique for implementing the new SI definition of the unit of mass. Metrologia, 53(5):A46-A74, sep 2016. 10.1088/0026-1394/53/5/a46.

[11] N. Rogge, J. Schleichert und C. Rothleitner. Investigations on motion deviations of an emfc balance. In Engineering for a Changing World: Proceedings. 59th IWK, Ilmenau Scientic Colloquium, Technische Universität IImenau, 2017.

[12] N. Rogge, S. Lin, C. Rothleitner und S. Vasilyan. Excitation frequency dependent deviations during the "velocity mode" of bl measurements in the planck-balance. In Proc. Int. Conf. on 23rd IMEKO TC4 International Symposium, Xi'an, China. IMEKO, 2019.

[13] N. Rogge, C. Rothleitner, S. Vasilyan, T. Fröhlich, F. Härtig und D. Knopf. Error sources in the force mode of the "pb2" planck-balance. Acta IMEKO, 9(5):53-57, dec 2020. 10.21014/acta_imeko.v9i5.958.

[14] Die Basis Serie OEM Wägezelle WZA215-LC. Sartorius Lab Instruments GmbH \& Co. KG, 2 2021. URL https: //www.sartorius.com/shop/ww/de/eur/anwendungen-laborwägen-oem-wägezellen/die-basis-serie-210-g-x-0-01-mg/p/ WZA215-LC.

[15] S. Schlamminger. Design of the Permanent-Magnet System for NIST-4. IEEE Transactions on Instrumentation and Measurement, 62:1524-1530, 2013. 10.1109/TIM.2012.2230771.

[16] SP 5000 DI Produktinformation. SIOS Messtechnik GmbH, 4 2019. URL https://sios.de/wp-content/uploads/2019/05/ SIOS_SP_DI_dt_kl.pdf.

[17] Tactron Elektronik GmbH. Rf-suisse, 2021. URL https: //www.tactron.de/hersteller/rf-suisse.html. RF Suisse RS-GGO10-O-GPSU.

[18] Y. Wang, N. Rogge, S. Vasilyan und T. Fröhlich. Investigations on nonlinear deformation phenomena in a force calibration system. Acta IMEKO, 9(5):150-155, dec 2020. 10.21014/acta_imeko.v9i5.958. 